\title{
CP AND T VIOLATION AT LONG BASELINE EXPERIMENTS
}

\author{
Sujata Diwakar ${ }^{1}$, Jyotsna Singh ${ }^{2}$, Yogita Pant ${ }^{3}$, R.B.Singh ${ }^{4}$, Alok Mishra ${ }^{5}$ \\ ${ }^{1}$ Department of Physics, University of Lucknow, Lucknow-226007, India \\ ${ }^{2}$ Department of Physics, University of Lucknow, Lucknow-226007, India \\ ${ }^{3}$ Department of Physics, University of Lucknow, Lucknow-226007, India \\ ${ }^{4}$ Department of Physics, University of Lucknow, Lucknow-226007, India \\ ${ }^{5}$ Department of Applied Science, Ambalika Institute of Management and Technology, Lucknow, India
}

\begin{abstract}
Existing Universe is matter dominated. Baryonic matter and leptonic matter asymmetry is one of the profound mystries in particle physics. The CP violation can elucidate the above mystery. Extent of bound, neutrino experiments can impose on CP violation will enlighten the path of particle physicist to go beyond the standard model. The matter effect in itself give rise to CP variance therefore looking for T violation will reinforce our predictions. Atmospheric neutrino mass difference is found to be around $10^{-3} \sim$ $10^{-2} \mathrm{eV}^{2}$. In order to make meaningful predictions with such small $\Delta \mathrm{m}^{2}$, baseline of the order of $1000 \mathrm{Km}$ to $100 \mathrm{Km}$ for a neutrino of energy $1 \mathrm{GeV}$ is required. In this work we have studied the menifestation of $C P$ and $T$ violation with the help of different proposed long baseline experiments for three neutrino framework.
\end{abstract}

\section{INTRODUCTION}

Lorentz symmetry and CPT symmetry are indispensible symmetries of standard model and general relativity. These fundamental symmetries do not hold at very high energies such as plank's scale therefore a vital question has erupted in science community whether Lorentz and CPT symmetries are violated at lower observable energies as well. We can think of plank supressed effects of these violations at observable energies and may attempt to observe them with the new high precesion experiments. Experimental validation of violation of such symmetries will give us signal to go beyond standard model or a need of new physics. Standard model (effective quantum field theory) and general relativity have been rigrously tested for years and no marked violation in them till date is observed. But standard model in its limitation, is not able to describe neutrino oscillations, Baryon asymmetry, Dark matter and quantum gravity. In an attempt to make this model complete a simple extension of standard model was required.The Standard model extension(SME) carries the essence of standard model along with neutrino mass, lorentz and CPT violating terms.

\section{THREE NEUTRINO}

OSCILLATION

\section{FRAMEWORK}

In three neutrino scheme the mixing matrix between the flavor eigen states $v_{\alpha}(\alpha=\mathrm{e}, \mu, \tau)$ and the mass eigenstates $v_{\mathrm{i}}$ (i $=1,2,3$ ) can be related as follows

$$
\left|\nu_{\alpha}>=\sum_{i} U_{\alpha i}\right| \nu_{i}>
$$

where $\mathrm{U}_{\mathrm{\alpha i}}$ is $3 \times 3$ unitary matrix known as PontecorvoMaki-Nakagawa-Sakata (PMNS) mixing matrix. In general case of $\mathrm{n}$ flavors, the mixing matrix $\mathrm{U}_{\alpha \mathrm{i}}$ depends on $\mathrm{n}$ mixing angles between the flavours $\left(\theta_{\mathrm{ij}}\right),(\mathrm{n}-1)(\mathrm{n}-2) / 2$ Dirac CP phases $\left(\delta_{\mathrm{CP}}\right)$ and $(\mathrm{n}-1)$ additional Majorana CP-violating phases. Three flavour lepton mixing matrix can be given as as

$$
U=U_{23} U_{13} U_{12} D=V D
$$

where

$$
\begin{gathered}
U_{12}=\left(\begin{array}{ccc}
c_{12} & s_{12} & 0 \\
-s_{12} & c_{12} & 0 \\
0 & 0 & 1
\end{array}\right) \\
U_{13}=\left(\begin{array}{ccc}
c_{13} & 0 & s_{13} e^{-i \delta} \\
0 & 1 & 0 \\
-s_{13} e^{i \delta} & 0 & c_{13}
\end{array}\right) \\
U_{23}=\left(\begin{array}{ccc}
1 & 0 & 0 \\
0 & c_{23} & s_{23} \\
0 & -s_{23} & c_{23}
\end{array}\right)
\end{gathered}
$$

and $\mathrm{D}=\operatorname{diag}\left(\mathrm{e}^{-{ }^{\mathrm{i} \varphi 1}}, 1, \mathrm{e}^{-\mathrm{i} \varphi 2}\right)$. The phase $\delta$ in $\mathrm{U}_{13}$ mixing matrix is Dirac-type CP violating phase, whereas the phases $\varphi_{1}$ and $\varphi_{2}$ are Majorana-type CP violating phases. Since majorana phases are common to all three flavors, they have no effect on neutrino oscillation. Therefore neglecting majorana phases, we get $\mathrm{U}=\mathrm{V}$. Now $3 \times 3$ neutrino mixing matrix also known as PMNS mixing matrix can be written as 


$$
\mathrm{U}=\left(\begin{array}{ccc}
c_{12} c_{13} & s_{12} c_{13} & s_{13} e^{-i \delta} \\
-s_{12} c_{23}-c_{12} s_{23} s_{13} e^{i \delta} & c_{12} c_{23}-s_{12} s_{23} s_{13} e^{i \delta} & s_{23} c_{13} \\
s_{12} s_{23}-c_{12} c_{23} s_{13} e^{i \delta} & -c_{12} s_{23}-s_{12} c_{23} s_{13} e^{i \delta} & c_{23} c_{13}
\end{array}\right)
$$

where $c_{i j}=\cos \theta_{i j}$ and $s_{i j}=\sin \theta_{i j}$.

Hamiltonian in vaccume is given by $\mathrm{H}$ and can be diagonalized with the help of PMNS mixing matrix (U).

$$
U^{\dagger} H U=\frac{1}{2 E}\left(\begin{array}{lll}
O & & \\
& \Delta m_{21}^{2} & \\
& & \Delta m_{31}^{2}
\end{array}\right)
$$

When the neutrinos pass through matter, an effective potential arises due to the presence of matter. Now the hamiltonian in matter can be written as

$$
\tilde{H}=H+\frac{1}{2 E}\left(\begin{array}{lll}
A & & \\
& 0 & \\
& & 0
\end{array}\right)
$$

where $\mathrm{A}=2 \mathrm{EV}_{\mathrm{cc}}=2 \sqrt{2} \mathrm{G}_{\mathrm{F}} \mathrm{N}_{\mathrm{e}} \mathrm{E}$ is the effective potential due to earth matter. $\mathrm{G}_{\mathrm{F}}$ is Fermi constant and $N_{e}$ is number density of electrons in earth matter. The matter potential A is proportional to energy $\mathrm{E}$ and therefore the second term in eq. (8) is energy dependent. The hamiltonian in matter is diagonalized by an effective PMNS matrix U

$$
\tilde{U}^{\dagger} \tilde{H} \tilde{U}=\frac{1}{2 E}\left(\begin{array}{lll}
\Lambda_{1} & & \\
& \Lambda_{2} & \\
& & \Lambda_{3}
\end{array}\right)
$$

where $\Lambda_{\text {is }}$ are mass eigen values in matter.Now the transition probability amplitude of neutrino in matter can be given by

$$
A\{(\alpha \rightarrow \beta): t\}=\sum_{i} U_{\alpha k}^{*} e^{-i \frac{\Lambda_{i}}{2 E} L} U_{\beta i}
$$

Probability equation for $v_{\alpha} \rightarrow v_{\beta}$ is given as

$$
\begin{gathered}
P_{\alpha \rightarrow \beta}=|A\{(\alpha \rightarrow \beta): t\}|^{2}(11) \\
P_{\alpha \rightarrow \beta}=\delta_{i j}-4 \sum_{i>j} \operatorname{Re}\left[\tilde{U}_{\alpha i} \tilde{U}_{\alpha j}^{*} \tilde{U}_{\beta i}^{*} \tilde{U}_{\beta j}\right] \sin ^{2} \Delta_{i j} \\
+2 \sum_{i>j} \operatorname{Im}\left[\tilde{U}_{\alpha i} \tilde{U}_{\alpha j}^{*} \tilde{U}_{\beta i}^{*} \tilde{U}_{\beta j}\right] \sin 2 \Delta_{i j}(12)
\end{gathered}
$$

The best fit values of neutrino oscillation parameters considered in our analysis are given in the Table 1.
Table 1: Best fit values of the neutrino oscillation parameters

\begin{tabular}{|c||c|}
\hline Parameters & best fit values \\
\hline$\theta_{12}$ & $34.4^{\circ}$ \\
\hline$\theta_{13}$ & $8.50^{\circ}$ \\
\hline$\theta_{23}$ & $45.0^{\circ}$ \\
\hline$\Delta m_{21}{ }^{2}$ & $8 \times 10^{-5} \mathrm{eV}^{2}$ \\
\hline$\Delta m_{31}{ }^{2}$ & $2.5 \times 10^{-3} \mathrm{eV}^{2}$ \\
\hline
\end{tabular}

\section{LONG BASELINE EXPERIMENT}

Amongst various proposed and ongoing neutrino oscillation experiments we have carefully selected five long baseline experiments for our studies. Long baseline experiments are best suited to study, CP violation and measurement of $\delta_{\mathrm{cp}}$ value of $\theta_{13}$ and the sign of $\Delta_{31}$. Recently Daya Bay [1] announced $\sin ^{2} \theta_{13}=0.090_{-0.009}^{+0.008}$ at $5 \sigma$ level and with this remarkable discovery the main focus of neutrino research has shifted to the discovery of other unknown senstive issues in neutrino sector like cp violation,octant degeneracy and mass hierarchy. Experiments used for our analysis are as follows
1) ICARUS
2) $\mathrm{T} 2 \mathrm{~K}$
3) MINOS
4) NOvA
5) NuFact

\subsection{ICARUS}

ICARUS experiment [2] [3] uses CNGS beam from CERN which falls at the detector, placed at Gran Sasso,730 km away from it. In this setup 470 ton Liquid Argon (LiAr) detector is taken as far detector where as no near detector is present in this setup. Its operational time is three years. The most prominant channel to probe neutrino oscillation parameter by ICARUS are $v_{\mu} \rightarrow v_{\mathrm{e}}$ and $\nu_{\mu} \rightarrow v_{\tau}$ appearance channels.

\subsection{T2K}

T2K was proposed in 2003 and it utilises $30 \mathrm{GeV}$ proton beam from J-PARC to generate the narrow band neutrino beam .T2K [4] was the first experiment to check the appearance of $v_{\mathrm{e}}$ from the $v_{\mu}$. T2K uses $22.5 \mathrm{Kt}$ Water cherenkov detector [5] far detector which is located $295 \mathrm{~km}$ away from the J-PARC. Its running time is taken to be 2 years with neutrino beam energy $\mathrm{E} \approx 1.3 \mathrm{GeV}$

\subsection{MINOS}

The MINOS (Main Injector Neutrino Oscillation Search experiment) [6] [7] uses proton beam of $120 \mathrm{GeV}$ from Fermilab, this beam produces a high intensity neutrino beam called NUMI beam. In this experiment two Magnetized Iron detectors (MID), used as near and far detectors are of masses 980 ton and $5.4 \mathrm{kt}$ respectively, these detectors are 
placed at $1 \mathrm{~km}$ and $735 \mathrm{~km}$ respectively from the beam source

\subsection{NOvA}

An off-axis neutrino beam $(\mathrm{E}=3 \mathrm{Gev})$ from Fermilab is used by NOvA experiment [8]. Which was especially designed to observe $v_{\mu} \rightarrow v_{\mathrm{e}}$ oscillation channel [9]. Another goals of NOvA experiment are to observe neutrino mass hierarchy,cp violation, $\theta_{23}$ octant. Totally Active Scintillation detectors (TASD) are used as near and far detectors with baselines 1 $\mathrm{km}$ and $812 \mathrm{~km}$ respectively from the source. The near detector is of $0.3 \mathrm{kt}$ while the far detector is of $25 \mathrm{kt}$.

\subsection{Neutrino Factory}

Neutrino factory is still in R \& D phase and is proposed to study the unknown parameters of neutrino oscillations. Neutrino factory will consist of a muon storage ring which will produce high intensity focussed muon beam [10] by decaying muons in its straight section [11] of the ring. The neutrino factory setup that we are considering here, consists of $10.66 \times 10^{20}$ muons of parent energy $50 \mathrm{GeV}$ that will decay in the muon storage ring. This experiment is designed to probe the $v_{\mu} \rightarrow v_{\mu}$ disappearance channel and $v_{\mathrm{e}} \rightarrow v_{\mu}$ appearance channel. In this setup, $50 \mathrm{Kt}$ Magnetized Iron detector (MID) detector is taken for our analysis and is placed at a distance of $3000 \mathrm{Km}$.

\section{CP AND T VIOLATION}

In this work we have observed the menifestations of $\mathrm{CP}$ and $\mathrm{T}$ violations [12] [13] [14] [15] in long baseline experiments. CPT being a fundamental symmetry in standard model indicates a peculiar relationship between neutrino and antineutrino oscillation probabilities which is expressed as $\mathrm{P}\left(v_{\alpha} \rightarrow v_{\beta}\right)=\mathrm{P}\left(\bar{v}_{\beta} \rightarrow \bar{v}_{\alpha}\right)$ where $\alpha, \beta=\mathrm{e}, \mu, \tau$. CPT invariance of any physical observable indicates that observable is symmetric under $\mathrm{C}, \mathrm{P}$ and $\mathrm{T}$ transformations or $\mathrm{CP}$ and $\mathrm{T}$ transformations respectively. If $\mathrm{CPT}$ is invariant then $\mathrm{CP}$ and $\mathrm{T}$ will be invariant, or the extent of violation in $\mathrm{CP}$ will be equal to the extent of violation in T. Matter in itself is asymmetric therefore matter effect gives rise to fake CPT and fake CP violations. When the neutrinos pass through matter, and $\Delta \mathrm{P}^{\mathrm{CP}} \neq 0$ is observed, then it does not necessarily means that $\mathrm{CP}$ symmetry is violated because even if $\delta_{\mathrm{CP}}(\mathrm{CP}$ phase $)$ is zero we can observe $\Delta \mathrm{P}^{\mathrm{CP}} \neq 0$ due to fake $\mathrm{CP}$ contribution that arises due to matter while $\Delta \mathrm{P}^{\mathrm{T}} \neq$ 0 will indicate a necessary violation in $\mathrm{T}$, irrespective of presence or absence of matter.

\section{A. CP Violation}

High precesion neutrino experiments can provide us a platform to study physics beyond standard model. In non standard physics, study of $\mathrm{CP}$ violation is very essential to explain baryon asymmetry of the Universe. The extent of CP odd violation can be examined by

$$
\Delta P^{C P}=P\left(\nu_{\alpha} \rightarrow \nu_{\beta}\right)-P\left(\bar{\nu}_{\alpha} \rightarrow \bar{\nu}_{\beta}\right)
$$

where $\alpha, \beta=\mathrm{e}, \mu, \tau$ and $\mathrm{P}\left(v_{\alpha} \rightarrow v_{\beta}\right)$ denotes the transition probability from a neutrino flavor $\alpha$ to a neutrino flavor $\beta$ and $\left(\bar{v}_{\alpha} \rightarrow \bar{v}_{\beta}\right)$ denotes the transition probability of anti neutrino flavor $\alpha$ to anti neutrino flavor $\beta$. The CP violating effects in neutrino oscillations can only be accessed in appearance channels. At given energies production and detection of $v_{\tau}^{\prime} \mathrm{s}$ is difficult, the oscillation channel $v_{\mu, \mathrm{e}} \rightarrow$ $v_{e, \mu}$ is considered as the most promising channel for the observance of experimental signatures of leptonic CP violation. Using time independent perturbation theory the expression for the oscillation probability $P\left(v_{\mu} \rightarrow v_{e}\right)$ in matter upto the second order in perturbative parameters $\lambda \equiv$ $\left|\Delta \mathrm{m}_{21}^{2}\right| /\left|\Delta \mathrm{m}^{2}{ }_{31}\right|$ and $\mathrm{s}_{13}$ is given as

$$
\begin{aligned}
P_{\mu c}= & 4 s_{13}^{2} s_{23}^{2} \frac{\sin ^{2}(\hat{A}-1) \Delta}{(\hat{A}-1)^{2}} \\
& +2 \lambda s_{13} \sin 2 \theta_{12} \sin 2 \theta_{23} \cos \Delta \cos \delta_{C P} \\
& \frac{\sin (\hat{A}-1) \Delta \sin \hat{A} \Delta}{\hat{A}-1} \\
& -2 \lambda s_{13} \sin 2 \theta_{12} \sin 2 \theta_{23} \sin \Delta \sin \delta_{C P} \\
& \frac{\sin (\hat{A}-1) \Delta \sin \hat{A} \Delta}{\hat{A}-1} \\
& +\lambda^{2} \sin ^{2} 2 \theta_{12} C_{23}^{2} \frac{\sin ^{2} \hat{A} \Delta}{\hat{A}^{2}}
\end{aligned}
$$

where $\hat{A}=\mathrm{A} / \Delta \mathrm{m}^{2}{ }_{31}=2 \sqrt{2} \mathrm{G}_{\mathrm{F}} \mathrm{N}_{\mathrm{e}} \mathrm{E} / \Delta \mathrm{m}^{2}{ }_{31}$ and $\Delta=\Delta \mathrm{m}^{2}{ }_{31}$ $\mathrm{L} / 4 \mathrm{E}$ and $\lambda \approx 0.033$. In more general form the oscillation probability $\mathrm{P}\left(v_{\mu} \rightarrow v_{\mathrm{e}}\right)$ can be written as

$$
\begin{gathered}
P_{\mu e}=P_{0}+P \cos \delta+P \sin \delta+P_{3} \\
P_{0}=4 s_{13}^{2} s_{23}^{2} \frac{\sin ^{2}(\hat{A}-1) \Delta}{(\hat{A}-1)^{2}} \\
P_{3}=\lambda^{2} \sin ^{2} 2 \theta_{12} c_{23}^{2} \frac{\sin ^{2} \hat{A} \Delta}{\hat{A}^{2}} \\
P_{\cos \delta}=2 \lambda s_{13} \sin 2 \theta_{12} \sin 2 \theta_{23} \cos \Delta \cos \delta_{C P} \\
\frac{\sin (\hat{A}-1) \Delta \sin \hat{A} \Delta}{\hat{A}-1} \\
P_{\sin \delta}=-2 \lambda s_{13} \sin 2 \theta_{12} \sin 2 \theta_{23} \sin \Delta \sin \delta_{C P} \\
\frac{\sin (\hat{A}-1) \Delta \sin \hat{A} \Delta}{\hat{A}-1}
\end{gathered}
$$

In the above expression term $\mathrm{P} \cos \delta$ is $\mathrm{CP}$ even or $\mathrm{CP}$ conserving term while $\mathrm{P} \sin \delta$ is $\mathrm{CP}$ odd or $\mathrm{CP}$ violating term as it switches its sign when we go from $P\left(v_{\mu} \rightarrow v_{e}\right)$ to 
$\mathrm{P}\left(\bar{v}_{\mu} \rightarrow \bar{v}_{\mathrm{e}}\right)$ channel. The probability expression for $\mathrm{P}\left(\bar{v}_{\mu}\right.$ $\rightarrow \bar{v}_{\mathrm{e}}$ ) channel is obtained by replacing $\delta_{\mathrm{CP}} \rightarrow-\delta_{\mathrm{CP}}$ and $\hat{A} \rightarrow$ $-\hat{A}$. As we know matter effect give rise to fake $\mathrm{CP}$ violation therefore keeping $\mathrm{A}<<1$

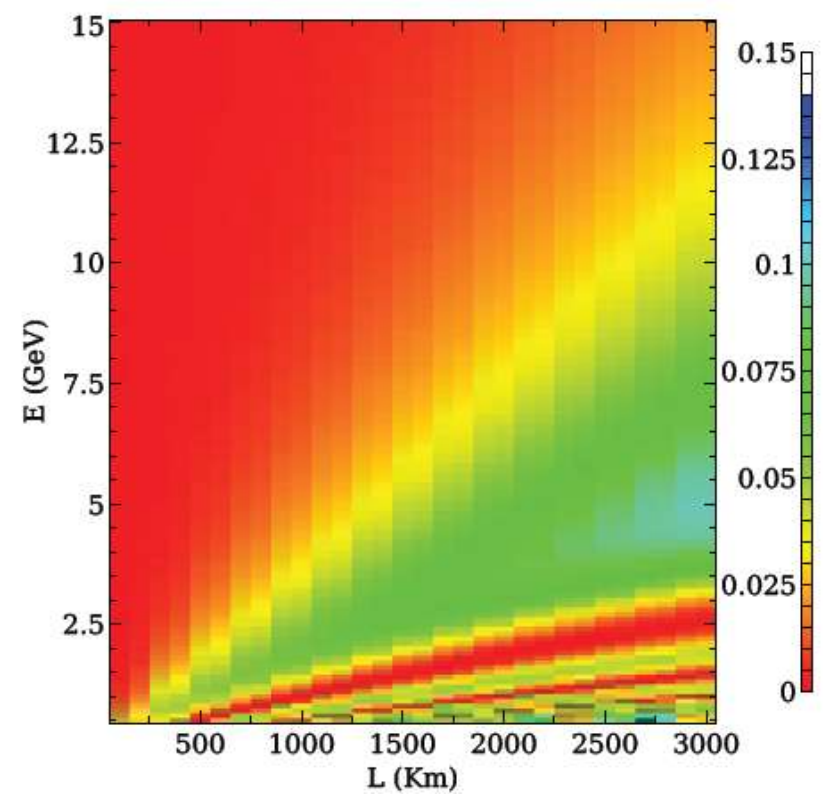

Fig. 1: $v_{\mu} \rightarrow v_{\mathrm{e}}$ transition probability oscillograph in energy and baseline plane with matter for normal hierarchy

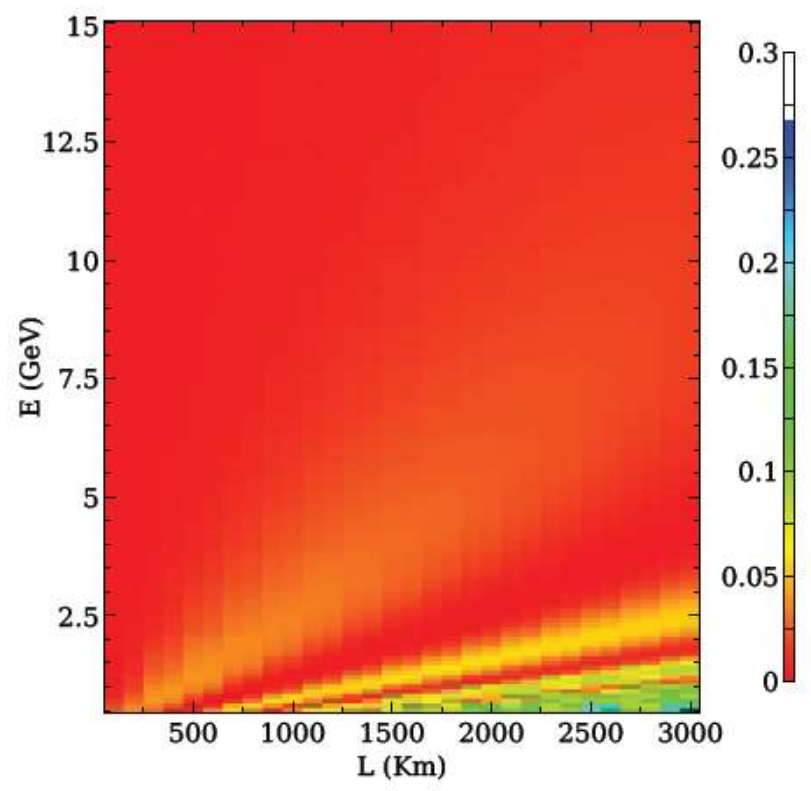

Fig. 2: $\bar{v}_{\mu} \rightarrow \bar{v}_{\mathrm{e}}$ transition probability oscillograph in energy and baseline plane with matter for normal hierarchy

(turning matter effects negligible ) in the equation (14), the equation is reduced as

$$
\begin{array}{r}
P_{\mu e}=\alpha^{2} \Delta^{2} \sin ^{2} 2 \theta_{12} c_{23}^{2}+4 s_{13}^{2} s_{23}^{2} \sin ^{2} \Delta \\
+\alpha \Delta s_{13} \sin 2 \theta_{12} \sin 2 \theta_{23}\left[\left(2 \Delta+\delta_{c p}\right)-\sin \delta_{\delta c p}\right]
\end{array}
$$

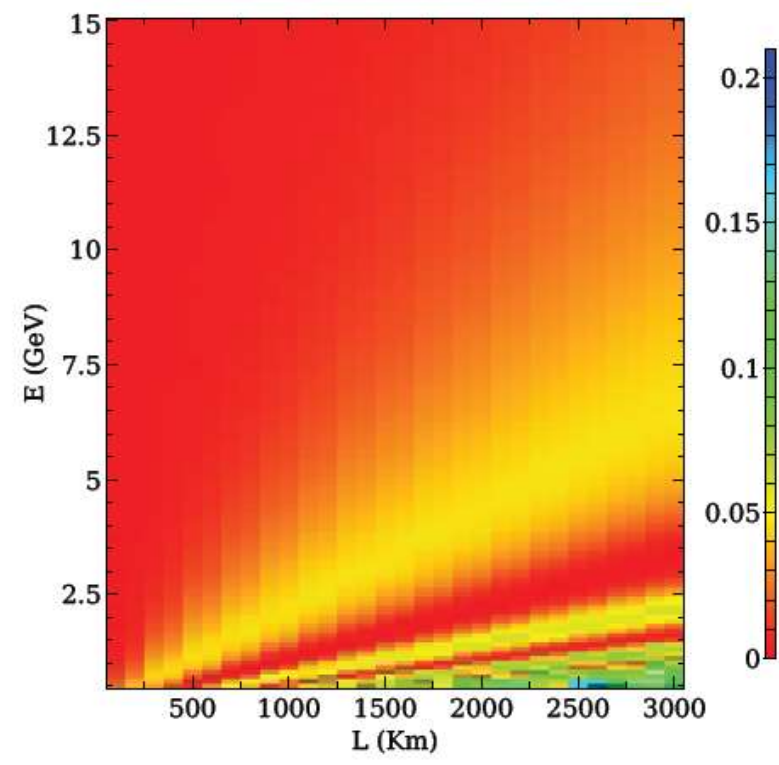

Fig. 3: $v_{\mu} \rightarrow v_{\mathrm{e}}$ transition probability oscillograph in energy and baseline plane, taking $\mathrm{A} \ll 1$ for normal hierarchy

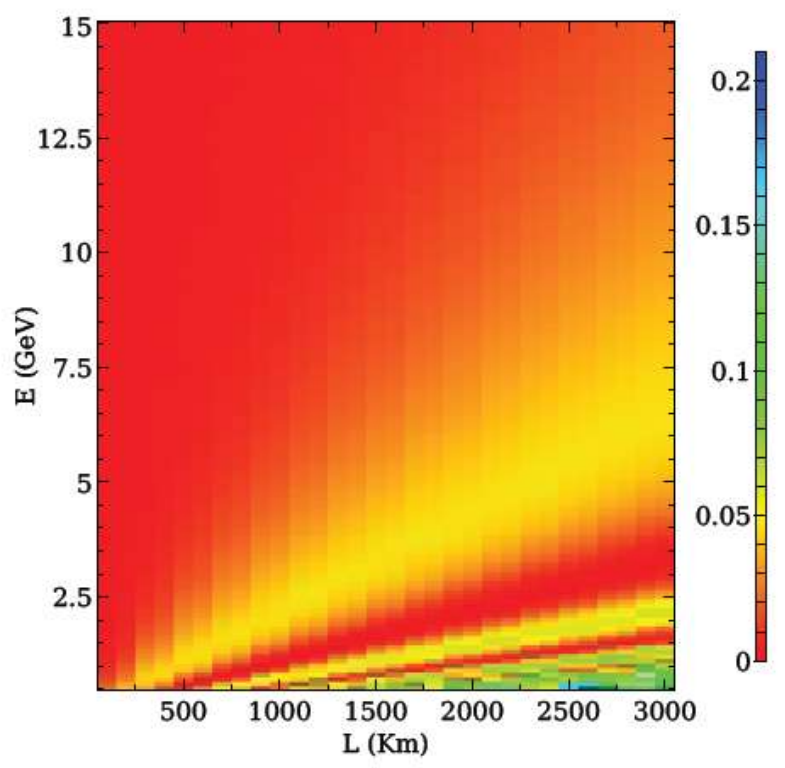

Fig. 4: $\bar{v}_{\mu} \rightarrow \bar{v}_{\mathrm{e}}$ transition probability oscillograph in energy and baseline plane, taking $A \ll 1$ for normal hierarchy

of Jarlskog factor given as

$$
J \approx \frac{1}{2} s_{13} \sin 2 \theta_{12} \sin 2 \theta_{23} \sin \delta_{C P} \approx 0.2 \sin \delta_{C P}
$$

CP asymmetry denoted by $A_{C P}$ helps in optimizing the baseline and energy for a given setup for the observance of $\mathrm{CP}$ violation.

Now the $\mathrm{CP}$ and $\mathrm{T}$ violation can also be define in terms 


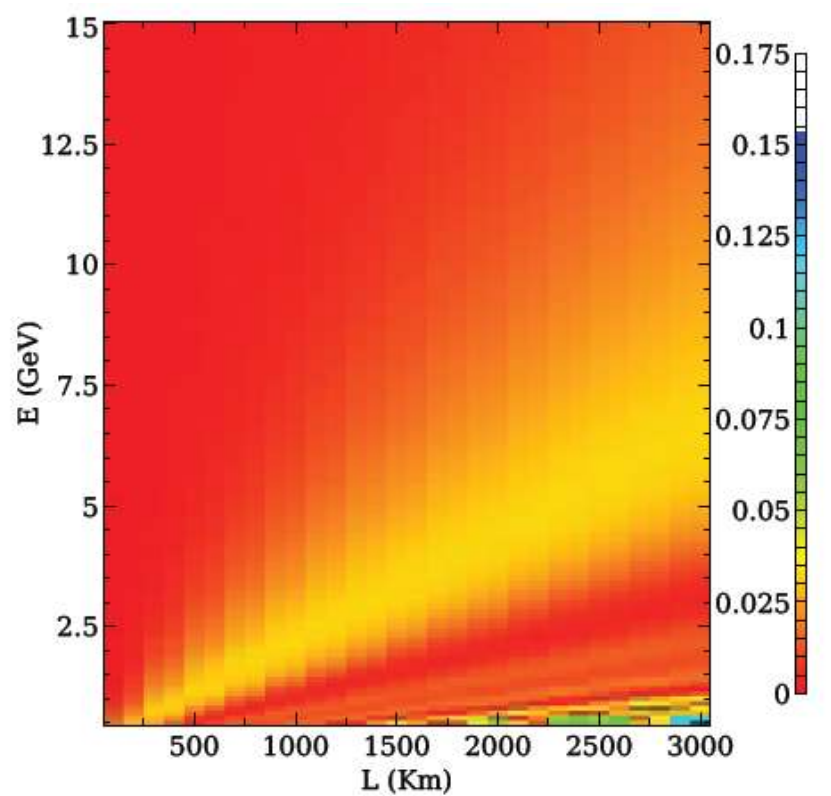

Fig. 5: $v_{\mu} \rightarrow v_{e}$ transition probability oscillograph in energy and baseline plane, taking $\mathrm{A} \ll 1$ at $\delta_{\mathrm{cp}}=\pi / 2$ for normal hierarchy

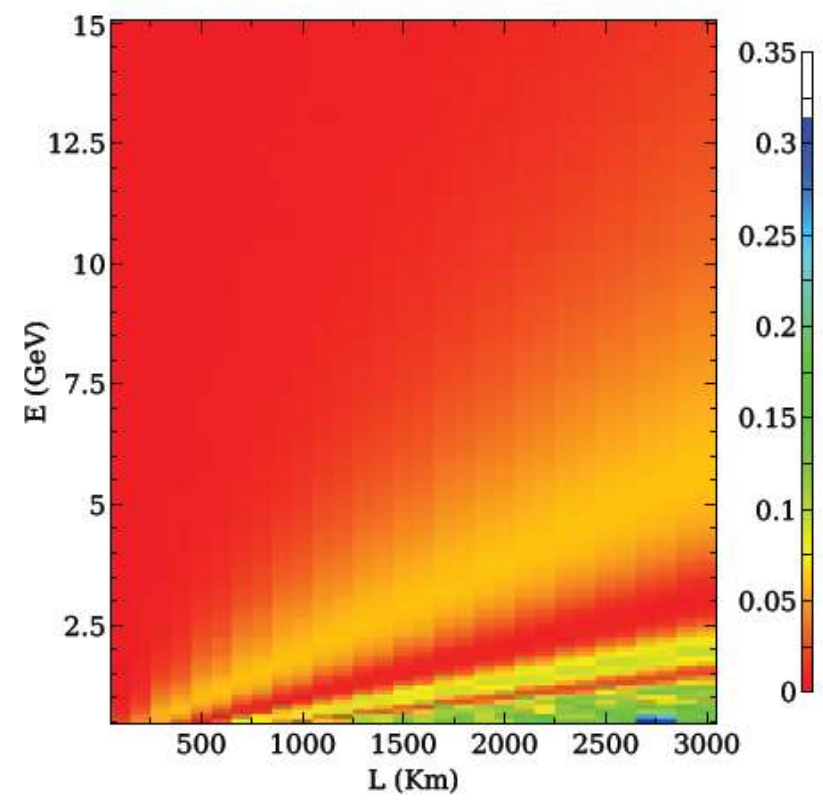

Fig. 6: $\bar{v}_{\mu} \rightarrow \bar{v}_{e}$ transition probability oscillograph in energy and baseline plane, taking $\mathrm{A} \ll 1$ at $\delta_{\mathrm{cp}}=\pi / 2$ for normal hierarchy

$$
A_{C P}=\frac{P\left(\nu_{\mu} \rightarrow \nu_{e}\right)-P\left(\bar{\nu}_{\mu} \rightarrow \bar{\nu}_{e}\right)}{P\left(\nu_{\mu} \rightarrow \nu_{e}\right)+P\left(\bar{\nu}_{\mu} \rightarrow \bar{\nu}_{e}\right)}
$$

Here we have observed the CP asymmetry at the probability level for different experiments, considering both mass hierarchies (normal and inverted).

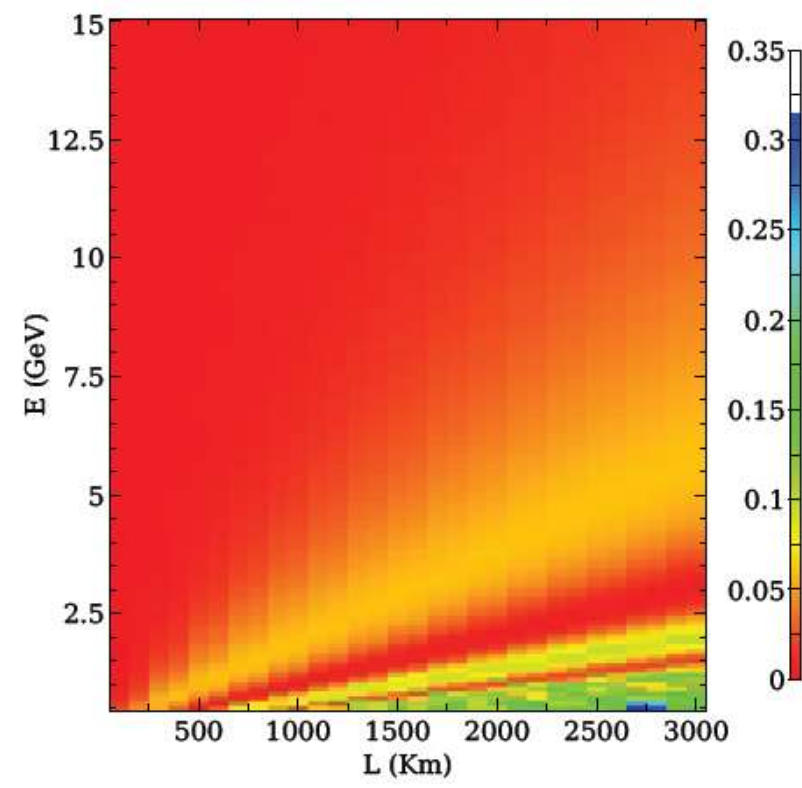

Fig. 7: $v_{\mu} \rightarrow v_{\mathrm{e}}$ transition probability oscillograph in energy and baseline plane, taking $\mathrm{A} \ll 1 \delta_{\mathrm{cp}}=\pi / 2$ for inverted hierarchy

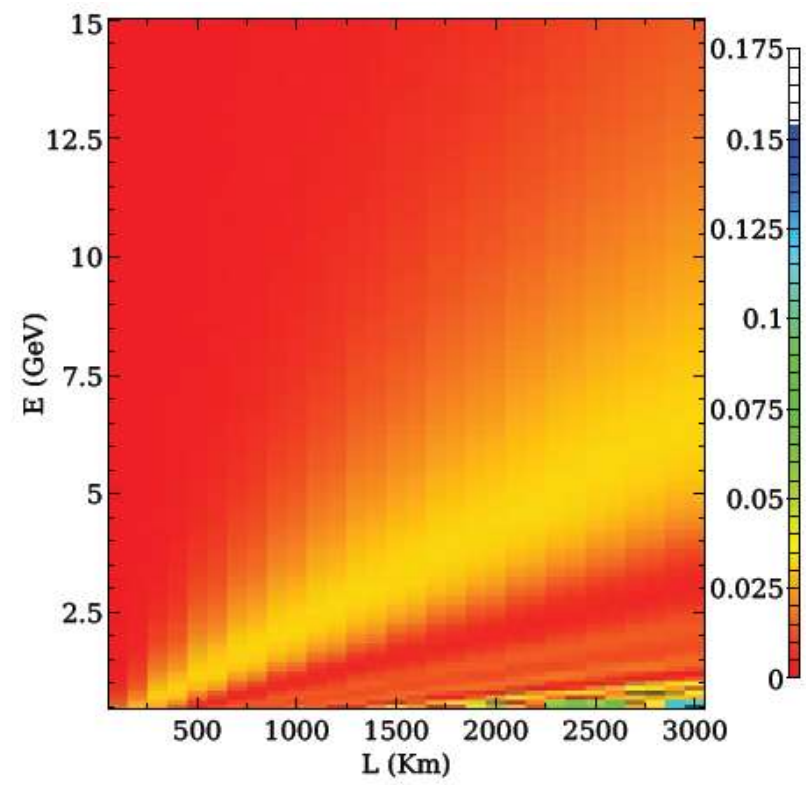

Fig. 8: $\bar{v}_{\mu} \rightarrow \bar{v}_{e}$ transition probability oscillograph in energy and baseline plane, taking $\mathrm{A} \ll 1$ at $\delta_{\mathrm{cp}}=\pi / 2$ for inverted hierarchy

After observing the $\mathrm{CP}$ violation trend at probability level, we have carried our analysis for five different long baseline setups at event level also. CP asymmetry at event level can be written as

$$
R_{C P}=\frac{N\left(\nu_{\mu} \rightarrow \nu_{e}\right)-N\left(\bar{\nu}_{\mu} \rightarrow \bar{\nu}_{e}\right)}{N\left(\nu_{\mu} \rightarrow \nu_{e}\right)+N\left(\bar{\nu}_{\mu} \rightarrow \bar{\nu}_{e}\right)}
$$




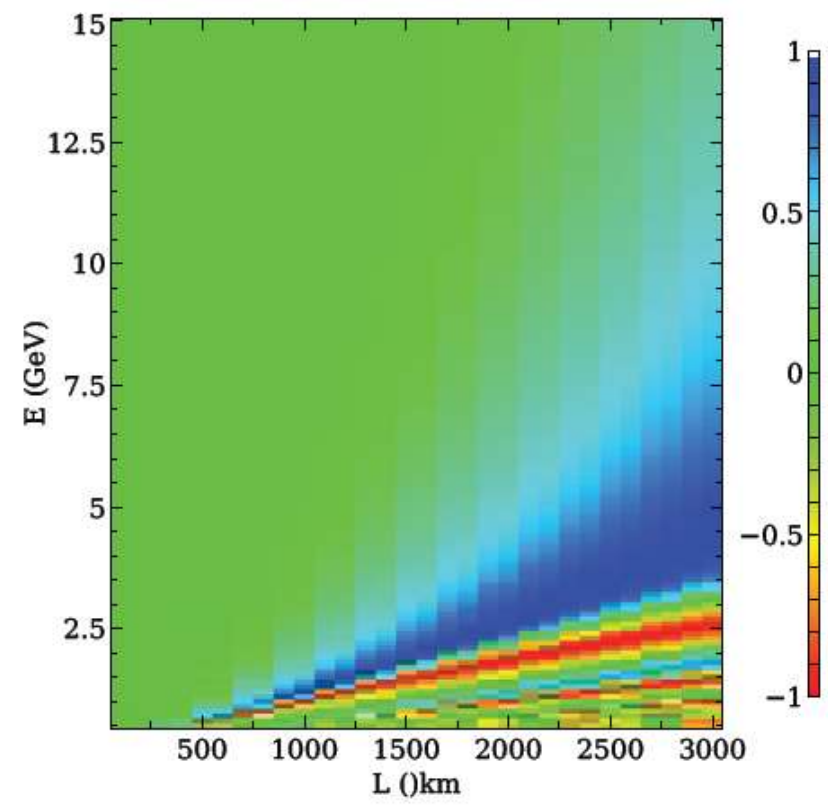

Fig. 9: Oscillograph for $A_{C P}(\mathrm{CP}$ asymmetry at probability level) in matter for $\delta_{\mathrm{cp}}=0$ in $\mathrm{L}$ and $\mathrm{E}$ plane for normal hierarchy

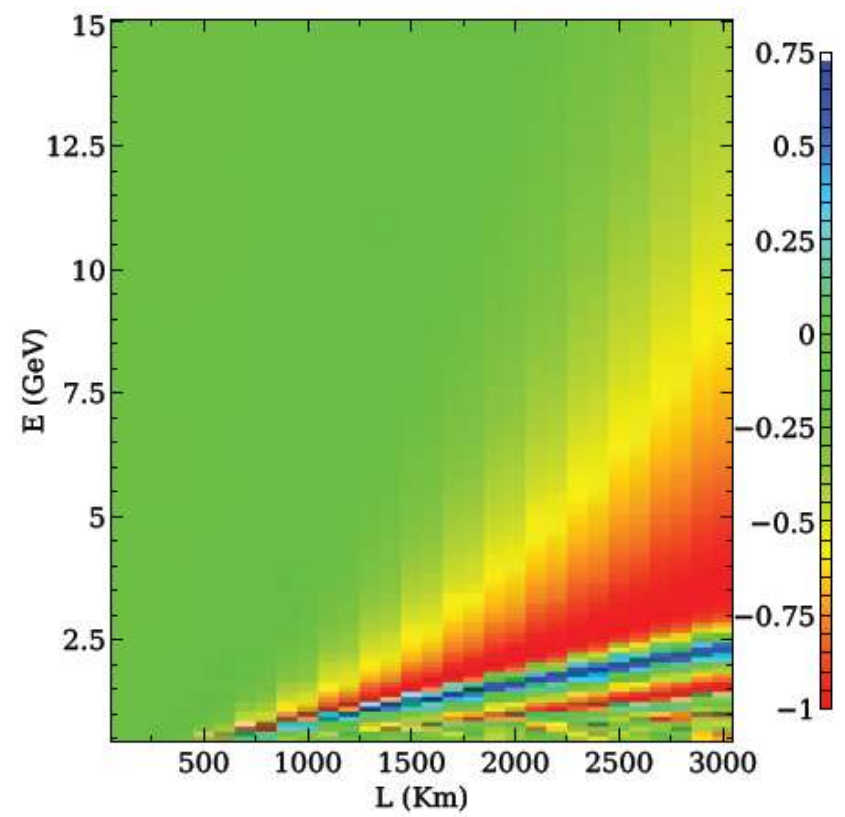

Fig. 10: Oscillograph for $A_{C P}(\mathrm{CP}$ asymmetry at probability level) in matter for $\delta_{\mathrm{cp}}=0$ in $\mathrm{L}$ and $\mathrm{E}$ plane for inverted hierarchy

where $\mathrm{N}$ denotes the number of events. In this work we have studied $R_{C P}$ for all five long baseline experiments for both hierarchies normal and inverted.

\subsection{T Violation}

When neutrinos pass through the matter, matter effects give rise to fake $\mathrm{CP}$ violation. Therefore

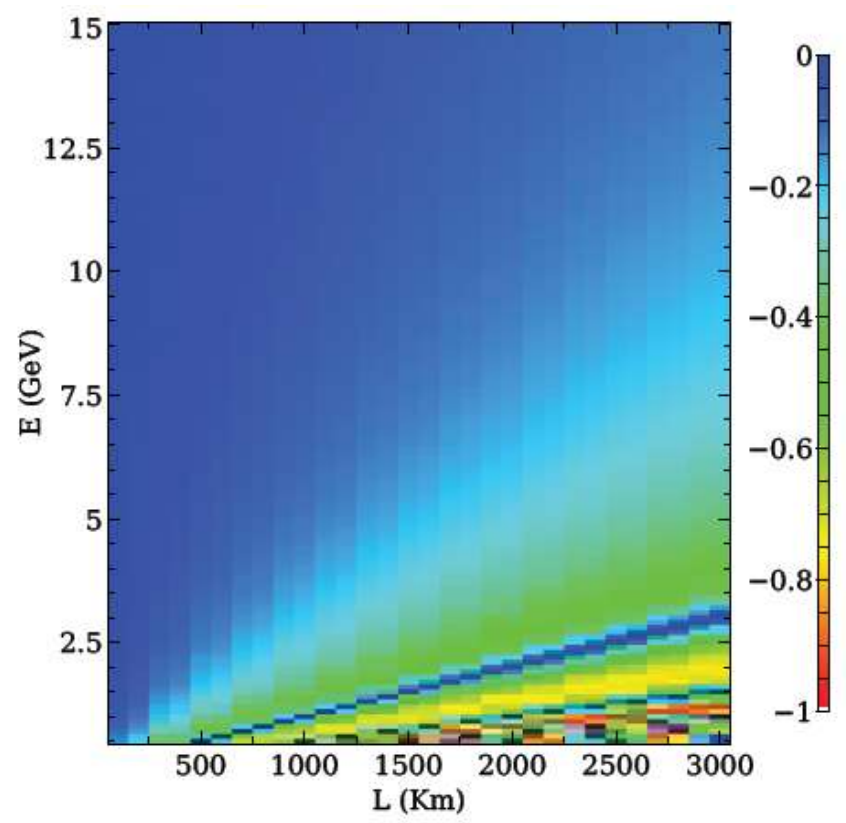

Fig. 11: Oscillograph for $A_{C P}$ (CP asymmetry at probability level), for $\mathrm{A} \ll<1$ for $\delta_{\mathrm{cp}}=\pi / 2$ in $\mathrm{L}$ and $\mathrm{E}$ plane for normal hierarchy
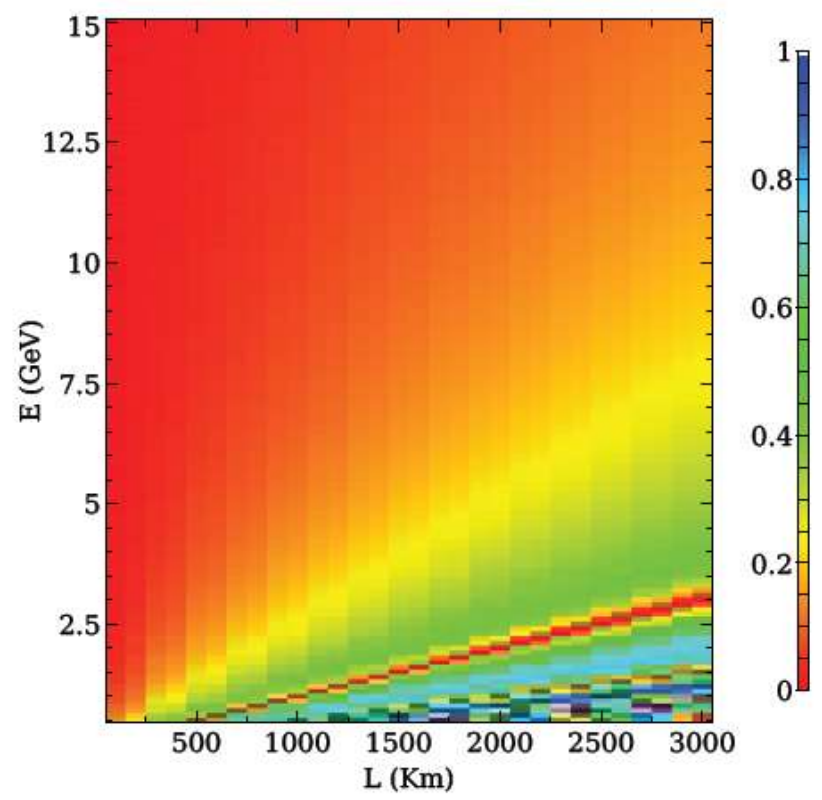

Fig. 12: Oscillograph for $A_{C P}(\mathrm{CP}$ asymmetry at probability level), for $\mathrm{A} \ll<1$ for $\delta_{\mathrm{cp}}=\pi / 2$ in $\mathrm{L}$ and $\mathrm{E}$ plane for inverted hierarchy

in presence of matter, study of $\mathrm{T}$ violation will be more reliable than study of $\mathrm{CP}$ violation. $\mathrm{T}$ violation at probability level can be written as

$$
\Delta P^{T}=P\left(\nu_{\alpha} \rightarrow \nu_{\beta}\right)-P\left(\nu_{\beta} \rightarrow \nu_{\alpha}\right)
$$

Transition probability $P_{\mu e}$ is given by eqn. (14). Substituting $\delta_{\mathrm{CP}}=-\delta_{\mathrm{CP}}$ in eq. 14 , we get transition probability for $\mathrm{P}_{\mathrm{e} \mu}$. 
For $v_{\mu} \rightarrow v_{\mathrm{e}}$ channel, $\mathrm{T}$ violation can be written as

$$
\begin{aligned}
\Delta P_{\mu e}^{T}= & \mathrm{P}_{\mu e}-P_{\psi \mu}=4 \lambda s_{13} \sin 2 \theta_{12} \sin 2 \theta_{23} \sin \Delta \sin \delta_{C P} \\
& \frac{\sin (A-1) \Delta \sin \hat{A} \Delta}{\hat{A}-1}
\end{aligned}
$$

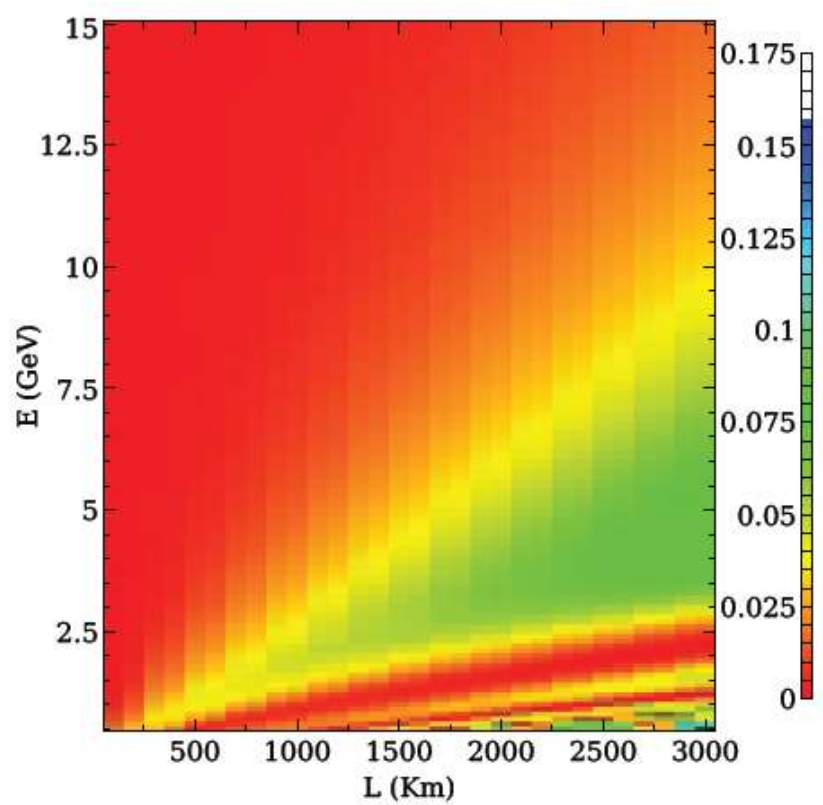

Fig. 13: Oscillograph showing $v_{\mu} \rightarrow v_{e}$ transition probability in energy and baseline plane for normal hierarchy

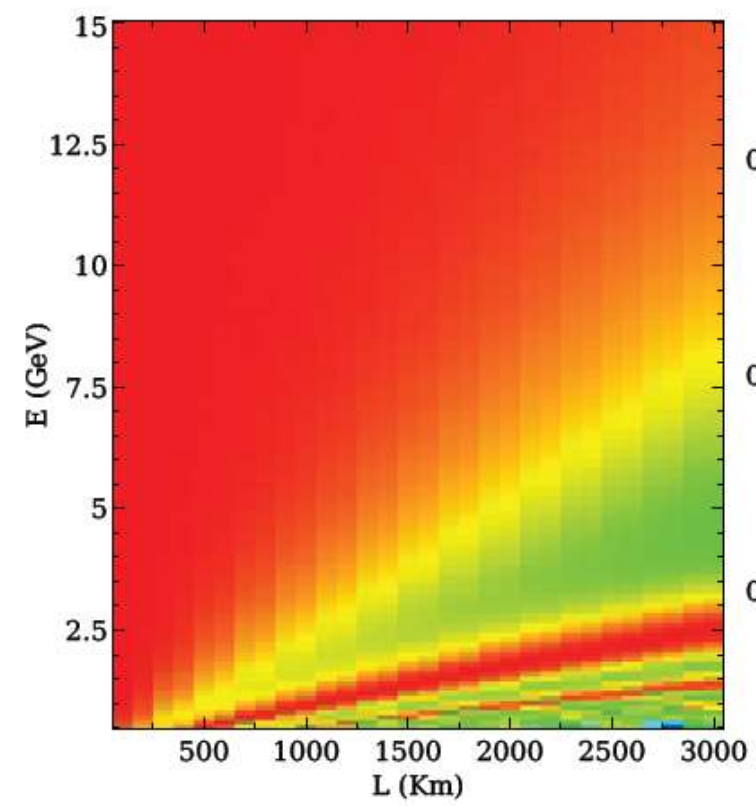

Fig. 14: Oscillograph showing $v_{e} \rightarrow v_{\mu}$ transition probability in energy and baseline plane for normal hierarchy

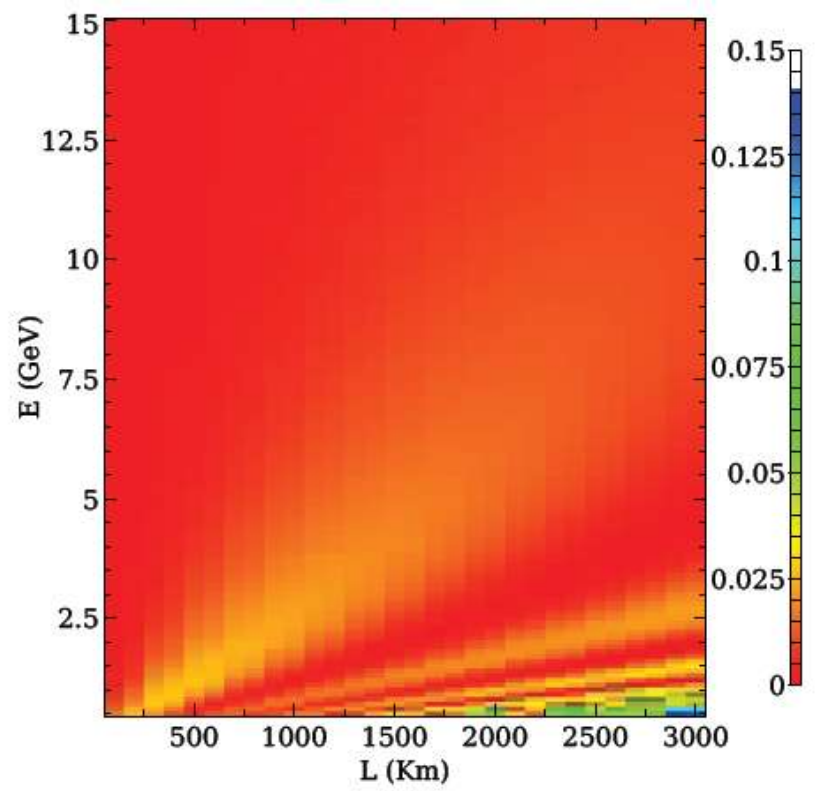

Fig. 15: Oscillograph showing $v_{\mu} \rightarrow v_{e}$ transition probability in energy and baseline plane for inverted hierarchy

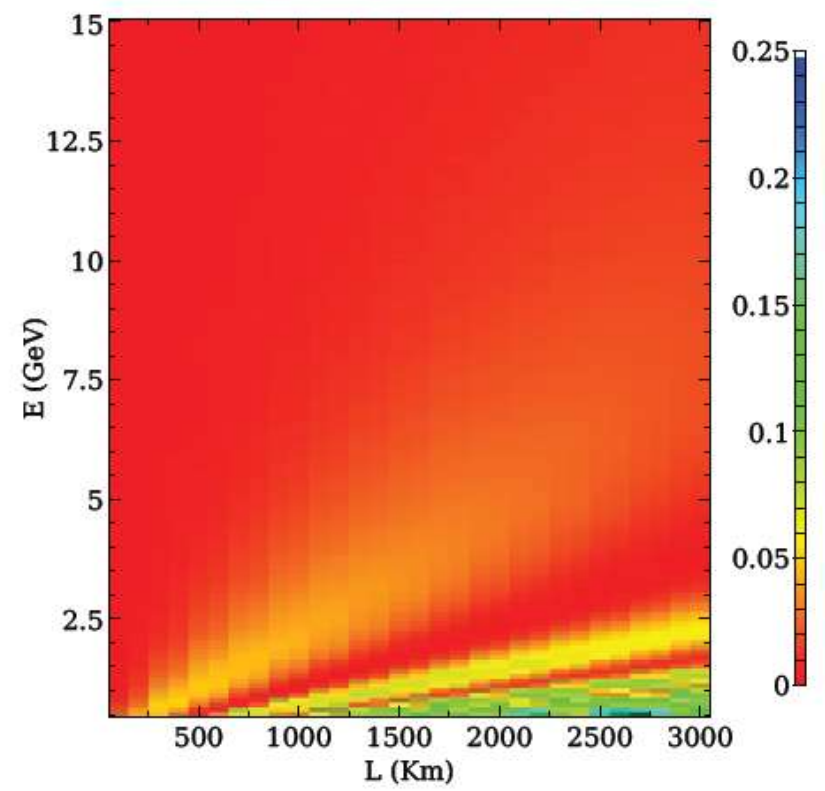

Fig. 16: Oscillograph showing $v_{e} \rightarrow v_{\mu}$ transition probability in energy and baseline plane for inverted hierarchy

Asymmetry factor at probability level can be expressed as

$$
A_{T}=\frac{P\left(\nu_{\mu} \rightarrow \nu_{e}\right)-P\left(\nu_{e} \rightarrow \nu_{\mu}\right)}{P\left(\nu_{\mu} \rightarrow \nu_{e}\right)+P\left(\nu_{e} \rightarrow \nu_{\mu}\right)}
$$

Variation in $\mathrm{T}$ asymmetry for different values of $\delta_{c p}$ is checked for all selected experiments for both hierarchies. 


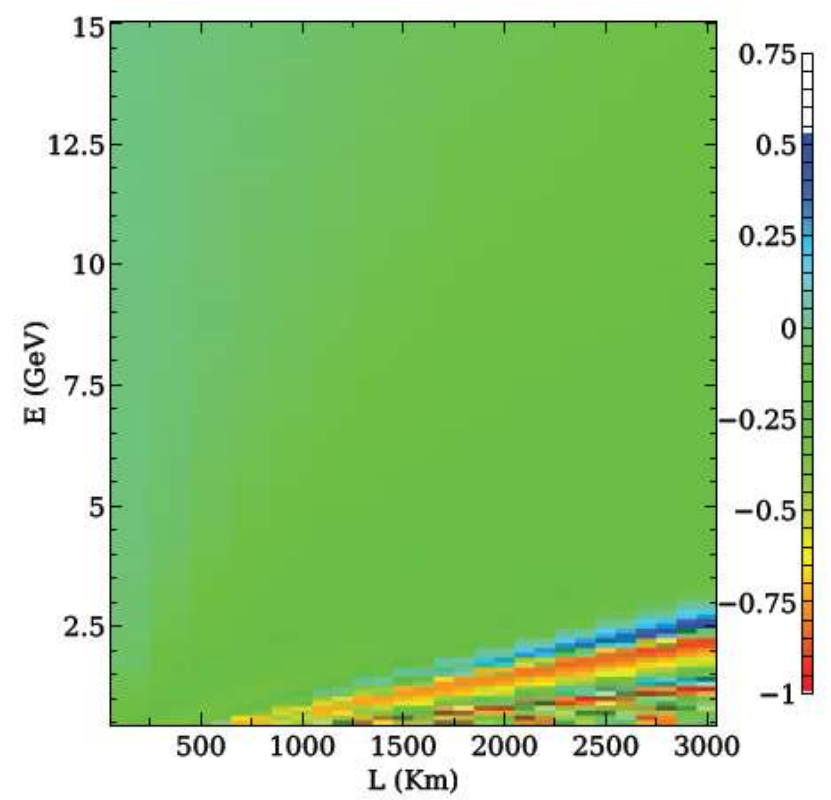

Fig. 17: Oscillograph for $A_{T}$ (T asymmetry at probability level) in $\mathrm{L}$ and $\mathrm{E}$ plane for normal hierarchy

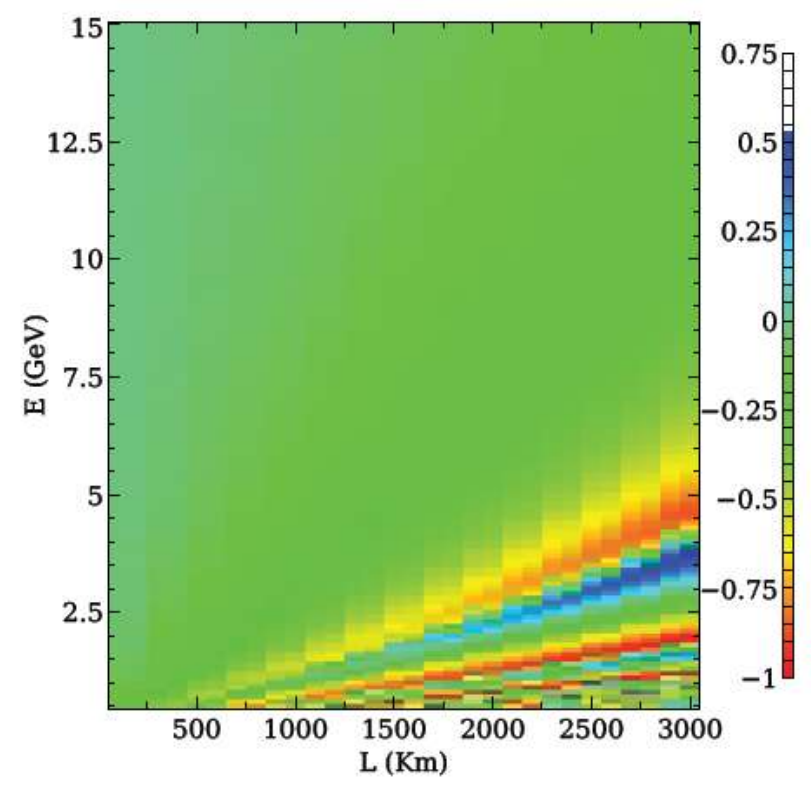

Fig. 18: Oscillograph for $A_{T}$ (T asymmetry at probability level) in $\mathrm{L}$ and $\mathrm{E}$ plane for inverted hierarchy

\section{SIMULATION SETUP FOR THE REVELATION OF CP AND T ASYMMETRY}

The simulation work for the revelation of $\mathrm{CP}$ and $\mathrm{T}$ violation in our analysis is done with the help of GLoBES [16].GLoBES, the Long Baseline Experiment Simulator [17] has two main Components

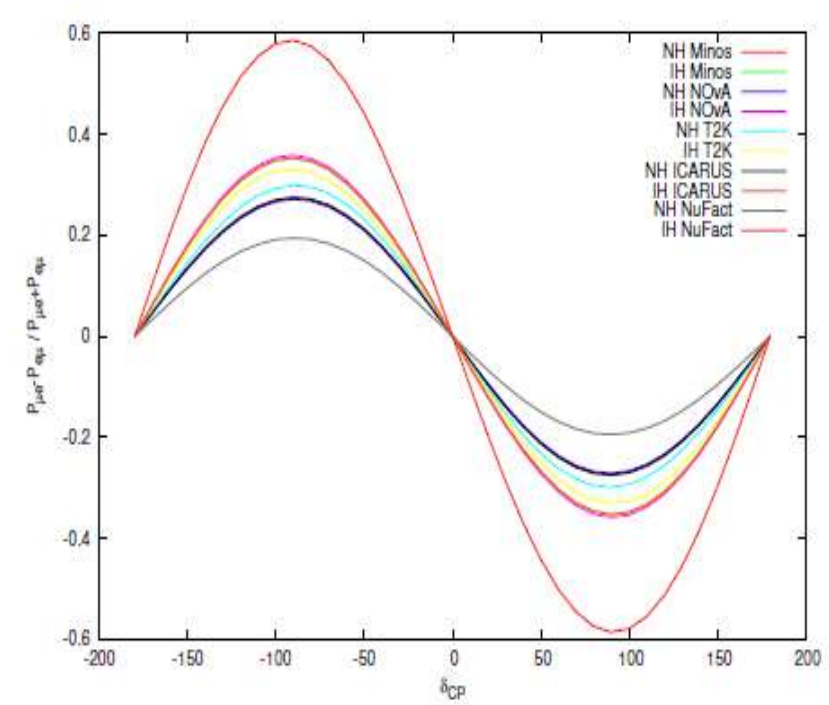

Fig. 19: T asymmetry with $\delta_{C P}$ at first oscillation node for different experiments in normal and inverted hierarchies.

1)AEDL, which describe individual experiments

2)User interface(C programs)

AEDL file contains type of source, energy used by the source beam, cross section, different properties of detector like efficiencies, backgrounds, energy resolution, systematics etc. In this work we have used different inbuilt AEDL files for the simulation purpose. Here we have defined the three neutrino flavor framework probability equation in the probability engine. After defining the probability, we are able to generate the oscillation probabilities for different desired channel with given set of oscillation parameters. In order to generate differential event rate we have included detector properties in the AEDL file. The differential event rate for each channel is given by

$$
\begin{array}{r}
\frac{d n_{\beta}^{I T}}{d E}=N \int_{0}^{\infty} \int_{0}^{\infty} d E d E \Phi_{\alpha}(E) \times \\
\frac{1}{L^{2}} P_{(\alpha \rightarrow \beta)}\left(E, L, \rho ; \theta_{12} \theta_{13} \theta_{23}, \Delta m_{31}^{2} \Delta m_{21}^{2}, \delta_{\varphi}\right) \times \\
\sigma_{f}^{I T}(E) k_{f}^{I T}(E-\hat{E}) \times \\
T_{f}(\hat{E}) V_{f}(\hat{E}-E)
\end{array}
$$

where

- $\alpha$ is initial flavor of neutrino

- $\beta$ is final flavor of neutrino

- $\Phi \alpha$ (E) flux of initial flavor at the source

- $\mathrm{L}$ is the baseline

- N normalization factor

- $\rho$ is matter density

- $\mathrm{E}$ is the incident neutrino energy

- $\hat{E}$ is energy of secondary particle

- $E^{\prime}$ is the measured neutrino energy as obtained from the experiment. 


\section{RESULTS AND DISCUSSION}

The results are given at probability and event level. The probability level analysis do not depend on detector properties, so we have checked these asymmetries at rate level also, As we know the mass hierarchy of neutrino is still not known hence in order to make our observations complete, we have checked the $\mathrm{CP}$ and $\mathrm{T}$ violation for both the hierarchies i.e. normal and inverted.

\subsection{Observation at Probability Level}

By looking at the oscillographs represented by Fig. 1 and Fig. 2, which shows transition probability $P\left(v_{\mu} \rightarrow v_{e}\right)$ and $P$ $\left(\bar{v}_{\mu} \rightarrow \bar{v}_{e}\right)$, we observe that the transition probability is maximum for one type of transition (neutrino mode) and minimum for the other transition (anti neutrino mode) for the same region in $\mathrm{L} \& \mathrm{E}$ plane. This behaviour gives a signal for CP violation, with matter effects. For $\mathrm{A} \ll 1$, both the transition probabilities $\left(P\left(v_{\mu} \rightarrow v_{e}\right) \& P\left(\bar{v}_{\mu} \rightarrow \bar{v}_{e}\right)\right.$ are same for the entire $\mathrm{L}$ and $\mathrm{E}$ range, this is shown by Fig. 3 and Fig. 4. This observation help us to conclude that there is no CP violation in this case when $A \ll 1$. Transition probabilities of neutrinos and antineutrinos can be checked keeping $\mathrm{A} \ll<1$ for different $\delta_{C P}$ values. This will give information related to extent of $\mathrm{CP}$ violation. The oscillographs shown by Fig (5), (6), (7), (8) are for $\delta_{C P}=90^{\circ}$ keeping A $\ll 1$ for both hierarchies. From above oscillograph this range can be pined down as 0.5 to $2.5 \mathrm{GeV}$ for $\mathrm{E}$ and 400 to $3000 \mathrm{Km}$ for L(baseline) .

Probability level asymmetry is observed for two different cases i.e. with matter effects and for $\mathrm{A} \ll 1$, taking $\delta_{\mathrm{CP}}$ value equals to zero. As from all the theoretical calculations we know that when the neutrino passes through asymmetrical matter, a fake CP asymmetry is introduced even if the dirac $\mathrm{CP}$ phase $\delta_{C P}$ is kept at zero .This can be observed by looking at asymmetry plots shown by Fig. (9) and Fig. (10). These plots are with matter effects and $\delta_{\mathrm{cp}}=0$.

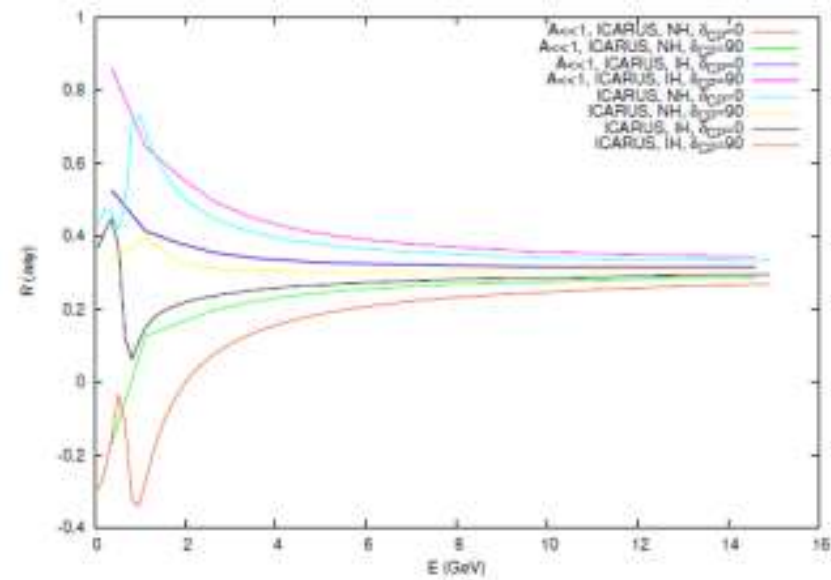

Fig. 20: For ICARUS experiment CP asymmetry $\left(R_{c p}\right) \mathrm{Vs}$ $\mathrm{E}(\mathrm{GeV})$ for two values of $\delta_{\mathrm{cp}}=\pi / 2$ and $\delta_{\mathrm{cp}}=0$ for both the hierarchies. Plots are for $\mathrm{CP}$ asymmetry with matter effects and for $\mathrm{A}<<1$

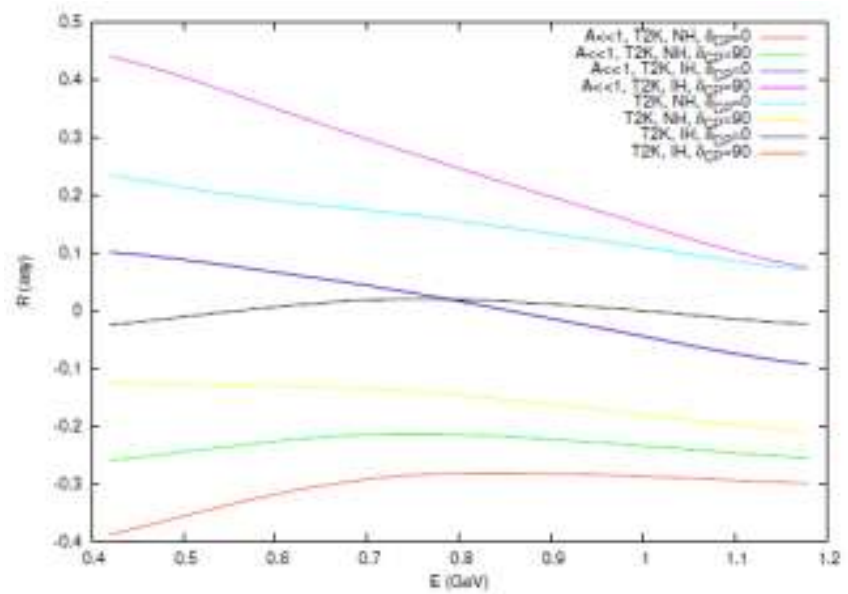

Fig. 21: For T2K experiment CP asymmetry $\left(R_{c p}\right) \mathrm{Vs}$ $\mathrm{E}(\mathrm{GeV})$ for two values of $\delta_{\mathrm{cp}}=\pi / 2$ and $\delta_{\mathrm{cp}}=0$ for both the hierarchies. Plots are for $\mathrm{CP}$ asymmetry with matter effects and for $\mathrm{A} \ll<1$

On the other hand for $\mathrm{A} \ll 1$ and $\delta_{\mathrm{CP}}=0$ we observe no asymmetry at the probability level, it indicates the absence of fake CP violation. Keeping this in mind we did our analysis for $\mathrm{A} \ll 1$ and $\delta_{\mathrm{CP}}=90^{\circ}$ in order to get the maximum CP manifestation at probability level. This can be observed by looking at plots shown by Fig. (11) and Fig. (12) which are for normal and inverted hierarchies respectively.

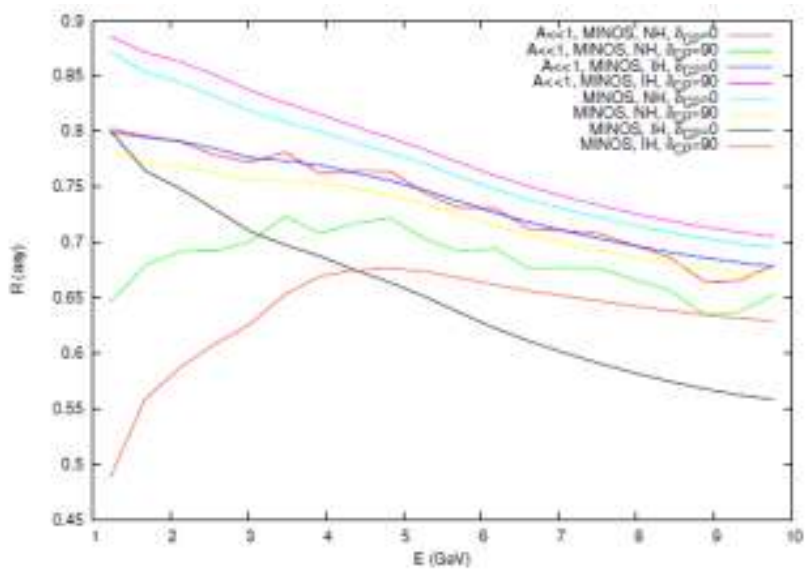

Fig. 22: For MINOS experiment CP asymmetry $\left(\mathrm{R}_{\mathrm{cp}}\right) \mathrm{Vs}$ $\mathrm{E}(\mathrm{GeV})$ for two values of $\delta_{\mathrm{cp}}=\pi / 2$ and $\delta_{\mathrm{cp}}=0$ for both the hierarchies.Plots are for $\mathrm{CP}$ asymmetry with matter effects and for $\mathrm{A} \ll 1$ 


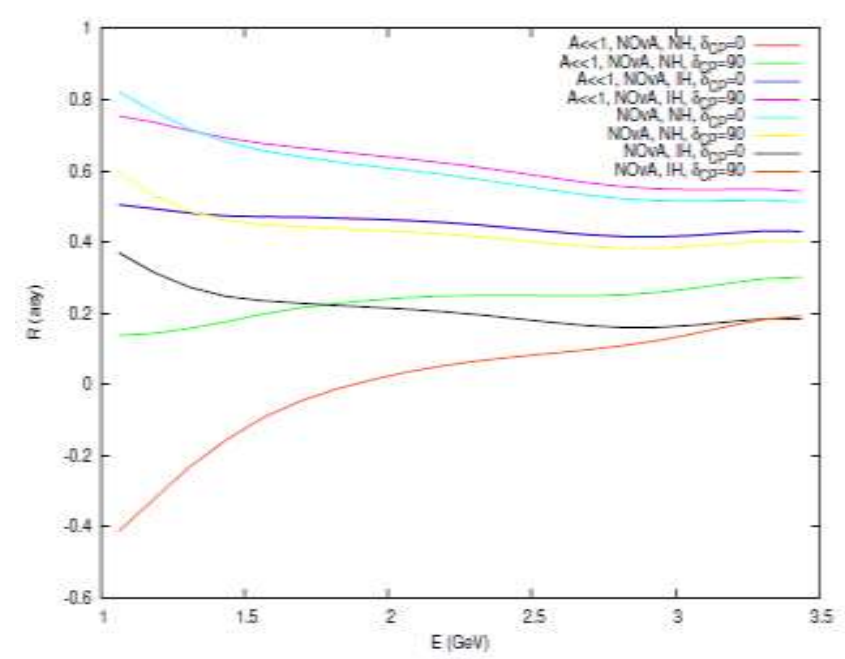

Fig.23: For NOvA experiment CP asymmetry $\left(R_{c p}\right)$ Vs $\mathrm{E}(\mathrm{GeV})$ for two values of $\delta_{\mathrm{cp}}=\pi / 2$ and $\delta_{\mathrm{cp}}=0$ for both the hierarchies.Plots are for $\mathrm{CP}$ asymmetry with matter effects and for $\mathrm{A}<<1$

Maximum asymmetry is revealed at lower energy and longer baseline .

Fig. (13),(14),(15),(16) are the probability oscillograph to check the $\mathrm{T}$ violation.By observing these oscillographs we see that there is a very narrow overlapping region of $\mathrm{E}$ and $\mathrm{L}$ for which transition probabilities, $\mathrm{P}\left(v_{\mu} \rightarrow v_{\mathrm{e}}\right)$ is maximum and $\mathrm{P}\left(v_{\mathrm{e}} \rightarrow v_{\mu}\right)$ is minimum or the other way round. To give more precise predictions at probability level for $\mathrm{T}$

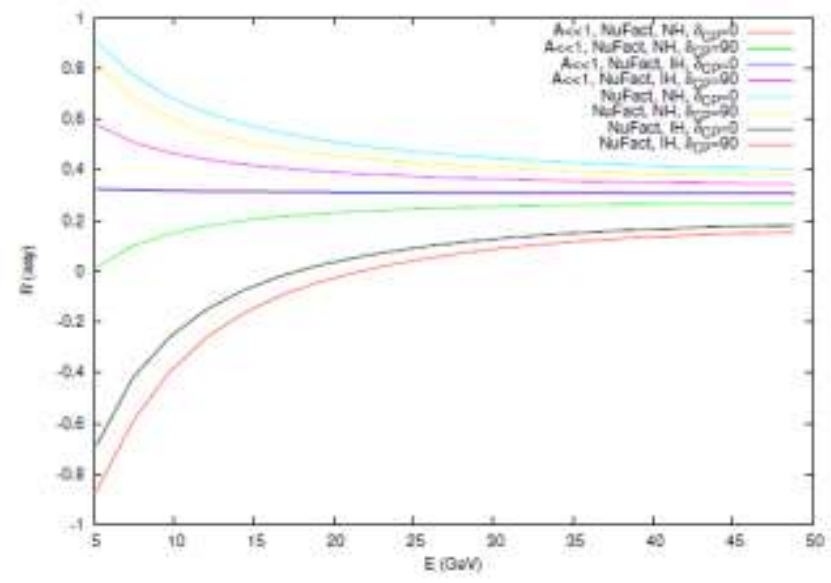

Fig. 24: For Neutrino Factory experiment $\mathrm{CP}$ asymmetry $\left(R_{\mathrm{cp}}\right) \mathrm{Vs} \mathrm{E}(\mathrm{GeV})$ for two values of $\delta_{\mathrm{cp}}=\pi / 2$ and $\delta_{\mathrm{cp}}=0$ for both the hierarchies.Plots are for CP asymmetry with matter effects and for $\mathrm{A} \ll<1$

violation we look at asymmetry plots given by Fig. (17) and (18) for both normal and inverted hierarchies respectively. Inverted hierarchy asymmetry plot exhibits better $\mathrm{T}$ asymmetry. T asymmetry can be observed for low energies and long baselines as indicated by the asymmetry plots. Fig. 19 demonstares probability level asymmetry with the variation of $\delta_{\mathrm{CP}}$, here we witness that the results with neutrino factory setup for $\delta_{\mathrm{CP}}=90^{\circ}$ considering inverted hierarchy as true hierarchy illustrate $\mathrm{T}$ violation maximally.

\subsection{Observation at Event Level}

At event level we have analysed the $\mathrm{CP}$ violation for two values of $\delta_{\mathrm{cp}}$ i.e $0^{\circ}$ and $90^{\circ}$. Entire analysis is carried for both mass hierarchies (Normal and Inverted). The variation of rate asymmetry with $\mathrm{E}$ have been observed in two cases (i) A $<<1$, (ii) with matter effects. Both the cases are taken up simultaneously by different experimental setups. These results are shown by fig. (20), (21), (22), (23), (24). For a given setup we get different observation of rate asymmetry for matter effect $\mathrm{A}<<1$ and with matter effects for same value of $\delta_{\mathrm{cp}}$. The difference between the two will give information related to $\mathrm{CP}$ asymmetry and simultaneously it will help us in the selection of experimental setup for probing $\mathrm{CP}$ violation. Watching all the five plots we can say, ICARUS in its normal hierarchy mode gives the best menifestation for $\mathrm{CP}$ violation at energy about $0.8 \mathrm{GeV}$. Rest of the experiments are also well placed for observation of CP violation.

\section{CONCLUSION}

From the above plots we conclude that $\mathrm{CP}$ and $\mathrm{T}$ asymmetries are better menifested at lower energy values and long baselines. Long baseline experiments provides the essential platform for the observance of $\mathrm{CP}$ and $\mathrm{T}$ violation . In case of $\mathrm{CP}$ asymmetry, matter induced $\mathrm{CP}$ effects should be distangled from pure $\mathrm{CP}$ asymmetry. In our work this is achieved by substituting $\mathrm{A} \ll 1$ in neutrino transition probability equation. Different experiments placed at different baselines illustrates maximum $\mathrm{CP}$ violation for energy range 0.5 to $5.5 \mathrm{GeV}$, while maximum $\mathrm{T}$ violation is exhibited in case of IH for energy range 1.8 to $2 \mathrm{GeV}$ and 4.5 GeV to $5.5 \mathrm{GeV}$. ICARUS seems to be the strong candidate for witnessing $\mathrm{CP}$ violation with $\mathrm{NH}$ consideration. NuFact for IH hierarchy gives a better platform to witness $\mathrm{T}$ violation. All operational long baseline experiments along with future baseline experiments can lead us to a well define values of $\mathrm{L}$ and $\mathrm{E}$ where we can think of exploring dirac CP phase.

\section{ACKNOWLEDGMENTS}

For this work, Sujata Diwakar is thankful to University Grant Commission, India for giving financial support under the Rajiv Gandhi National Fellowship scheme and Jyotsna Singh acknowledge DST for sanctioning the project titled" R \& D efforts by university group for INO", with the project no. is SR/MF/PS-02/2013-Lucknow(c).

\section{REFERENCES}

[1]. F. An , et al. Spectral measurment of electron antineutrino oscillation amplitude and frequency at Daya Bay , Phys. Rev. Lett 112(2014)061801, arXiv:1310.6732 [2]. ICARUS collaboration,S Amerio et al.,Design,construction and tests of the ICARUS T600 detector,Nucl,Instrum.Meth A 527(2004)329

[3]. ICARUS collaboration,S Amerio et al.,study of electron recombination in liquid Argon with ICARUS TPS,Nucl. Instrum. Meth A 523(2004)275 
[4]. Abe K et al. (T2K collab.)Nucl Instrum. Meth. Phys. Rev A659

[5]. Abe K et al. (T2K collab.)Nucl Instrum. Meth. Phys. D $88032002(2013)$

[6]. Adamson et al.[MINOS Collaboration],Phys. Rev. Lett. 106,181801(2011),arxiv:1103.0340[hep-ex]

[7]. D. G Michael st al.[MINOS Collaboration],Phys. Rev. Lett. 97,191801(2006), hep-ex/0607088

[8]. Ayres D et al. (NOvA Collab.),arXiv:1307.7335

[9]. D.S Ayres et al. The NOvA Technical Design Report 2007.

[10]. ed Autin B, Blondel A, and Ellis J 1999 Prospective Study of Muon Storage Rings at CERN Report CERN 9902, ECFA 99-197 ed Holtkamp N and Finley D 2000 Feasibility Study of a Neutrino Source Based on a Muon Storage Ring Report FERMILAB-PUB-00/108-E

[11]. Geer S 1998 Phys. Rev. D 576989

[12]. M. Tanimoto, Phys. Rev. D 55, 322 (1997); J. Arafune and J. Sato, Phys.Rev. D55, (1997)

[13]. J. Arafune, M. Koike, and J. Sato, Phys. Rev. D 56, 3093 (1997); Erratum62, 119905 (1999)

[14]. H. Minakata and H. Nunokawa, Phys. Lett. B 413, 369 (1997); Phys. Rev.D 57, 4403 (1998).

[15]. M. Koike and J. Sato, Phys. Rev. D 61, 073012 (2000); Erratum 62, 079903(2000)

[16]. P. Huber, M. Lindner, and W. Winter, Comput. Phys. Commun. 167, 195 (2005),hep-ph/0407333

[17]. P. Huber, J. Kopp, M. Lindner, M. Rolinec, and W. Winter, Comput. Phys. Commun.177, 432 (2007),hep$\mathrm{ph} / 0701187$ 